\title{
NUCLEAR EXCITATIONS AND REACTION MECHANISMS
}

\section{Report of Progress}

1 November 1979 to 30 September 1980

This book was preanesed a DISCLAIMER

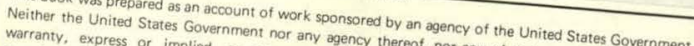

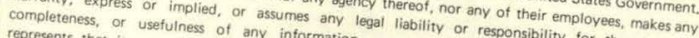
represents that its use wowld of any information, apparatus, predssonsibility for the accuracy.

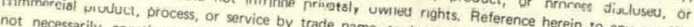

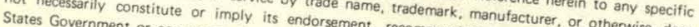
hecascartivm ment or any agency thereof The

Department of Physics

Brown University

Providence, Rhode Island

Contract DE-AC02-76ER03235.A004

1 October 1980

\section{MACTER}

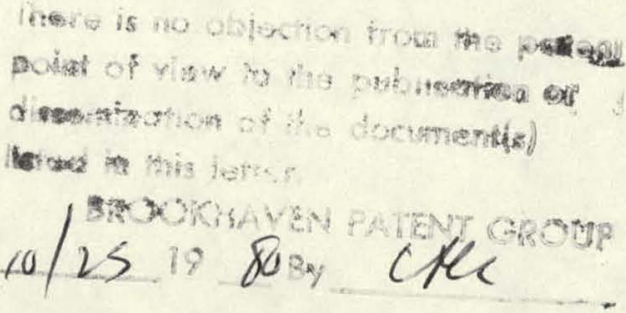




\section{DISCLAIMER}

This report was prepared as an account of work sponsored by an agency of the United States Government. Neither the United States Government nor any agency Thereof, nor any of their employees, makes any warranty, express or implied, or assumes any legal liability or responsibility for the accuracy, completeness, or usefulness of any information, apparatus, product, or process disclosed, or represents that its use would not infringe privately owned rights. Reference herein to any specific commercial product, process, or service by trade name, trademark, manufacturer, or otherwise does not necessarily constitute or imply its endorsement, recommendation, or favoring by the United States Government or any agency thereof. The views and opinions of authors expressed herein do not necessarily state or reflect those of the United States Government or any agency thereof. 


\section{DISCLAIMER}

Portions of this document may be illegible in electronic image products. Images are produced from the best available original document. 
"Th1s report was prepared as an account of Government-sponsored work. Neither the United States, not the Energy Research and Development Administration, nor any person acting on behalf of the Administration:

A. Makes any warranty or representation, expressed or implied, with respect to the accuracy, completeness, or usefulness of the information contained in this report, or that the use of any information, apparatus, method or process disclosed in this report may not infringe privately owned rights; or

B. Assumes any liabilities with respect to the use of, or for damages resulting from the use of, any information, apparatus, method, or process disclosed in this report.

As used in the above, 'person acting on behalf of the Administration' includes any employee or contractor of the Administration, or employee of such contractor, to the extent that such employee or contractor of the Administration, or employee of such contractor prepares, disseminates, or provides access to, any information pursuant to his employment or contract with the Administration, or his employment with such contractor." 


\section{UNCLASSIFIED}

\section{ABSTRACT}

A. (Fallieros). Photon scattering, consistency condition between seagull quadrupole terms and the absorption sum rule, Raman scattering to negative-parity states, non-local terms due to exchange and retardation effects in charge-transfer reactions.

B. (Levin). Consistency and meaning of various approximate channel coupling array equations; derivation of equations used in empirical nuclear reaction analyses; multi-cluster, n-particle scattering theory; computer code modifications; converged molecular bound state calculations; Ninth International Conference on the Few Body Problem:

C. (Li). Consistency of approximate channel coupling array equations; derivations of equations used in empirical nuclear reaction analyses; WKB-type approximation in angular momenta for central potentials. 


\section{CONTENTS}

1. Scope

2. Current Research:

A. (Prof. S. Fallieros) 2

B. (Prof. F. S. Levin) 8

C. (Dr. C. T.: Li) 15

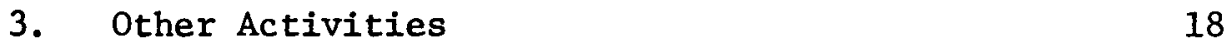

4. Personne1 22

5. Year's Publications 23 


\section{SCOPE}

The nuclear physics group at Brown University has continued to comprise five physicists, two faculty members in experimental physics and the two faculty members and one Research Associate engaged in theoretical research. As in the past, the collaboration between experimentalieto and theoriete continues.

This progress report contains in the three parts, A, B, and C, the descriptions of the work of Professors Fallieros and Levin and of Dr. Li. Research is carried out in the Barus and Holley Physics Engineering Building while numerical analyses have been done at Brown on the IBM System 370 . 


\section{CURRENT RESEARCH}

2A. (Fa11ieros)

Photon scattering and exchange contributions in the nuclear seagull term.

As is well known, the interaction of a photon, represented by a vector potential term $\vec{A}$, with a non-relativistic system contains two terms: one linear in $\vec{A}$ as well as in the particle-current density and a second quadratic in $\vec{A}$. The latter is the so-called seagull term and for a simple atomic system it represents a localized, point interaction involving two photons. In the case of a nucleus, charge-exchange interactions are known to produce a spreading of this term over a finite region. Its shape and strength are still unknown. In an effort to emphasize the usefulness and clarify the means of extracting information about this term, we have obtained some resuits which will be illustrated here in the case of quadrupole scattering of photons. We keep in mind that, the quadrupole absorption of photons is constrained by an energy-weighted sum rule. This sum rule contains an exchange component related to isovector excitations. In the scattering of photons, on the other hand, we have the two contributions mentioned above: the dispersive term (1inear in $\vec{A}$ ) and the seagu11 term. Both contain quadrupole components and both contain exchange effects. The dispersive term is also related, through its imaginary part, to the absorption cross section. In the analysis of a photon-scattering experiment we have to keep these contributions in mind and parametrize them appropriately in order to fit the data and extract physical information. The unknown nature of the seagul1 term would seem to present a dificulty, often resolved by an unjustifiable neglect of its exchange part. 
What we have emphasized is that it should be sufficient to consider quadrupole seagull terms up to quadratic in the photon energy as long as the latter is limited to $100-150 \mathrm{MeV}$ and that the coefficient representing exchange must be exactly the quantity that also appears in the quadrupole-absorption sum rule mentioned above. To demonstrate this, one relies on the observations that, at low energies, the dispersive term becomes proportional to the quadrupole sum rule and that in the complete amplitude at low energies, the quadrupole contributions first appear with the fourth (not the second) power of the photon energy. This last statement was first proved by Friar for the complete nuclear problem including exchange: It follows from the above that the quadratic seagull and dispersive terms just cancel each other and, therefore, the seagull is itself proportional to the energy-weighted quadrupole sum rule. This result, hopefully, clarifies the relation between scattering and absorption of photons and limits the number of unknown parameters needed in the analysis of an experiment. We may add that the statement concerning the energy-dependence of the quadrupole component is rather simple to summarize: at low energies, we can safely ignore retardation corrections to the quadrupole amplitude. In this case, however, we can (through a gauge transformation) combine the seagull and the dispersive terms into a single term. This term is essentially identical to the response function of the system to an external classical electric field whose amplitude contains the square of the photon frequency. Since the transition rate is the square of this amplitude, the fourth-power dependence, mentioned above, follows.

Negative-parity excitations in nuclear Raman scattering.

Our effort in this particular investigation has been rather limited. We have obtained some results which we describe below. We have found the work in 
this direction rather appealing and we expect to continue our study of this problem. The general form of the total transition amplitude has been written down in the relatively long-wavelength limit and for transitions of the $0^{+} \rightarrow 1^{-}$ type. As anticipated, the lowest-order contributions involve now transitions of electric and magnetic dipole or electric dipole and quadrupole character. The angular distribution typically contains the $\cos \theta$ and $\cos ^{3} \theta$ interference terms characteristic of these mixtures. Predictably, cancellations are found to occur as a result of the principle of gauge invariance. These cancellations both simplify the overall amplitude and impose constrains which should be useful for the interpretation of experimental results. Some detailed calculations have been performed for systems of independent particles. For atoms, the general indication is that the Raman cross section will be large when a corresponding dipole matrix element for a transition between initial and final states is also large. The same trend is also expected for the nucleus. In this case, however, the information contained in the Raman amplitude is complementary rather than essentially repetitive to that of a direct one-photon transition. This is because the interaction terms are now different and a comparison of matrix elements between the same initial states is quite informative. As is well known, the Raman transition matrix element is very similar to that of a two-photon decay of the final state. For states such as the $1^{-}$states considered above, however, the two-photon branching will be completely insignificant and cannot be of any physical interest. Another process, though, which might be of some interest is the Raman transition initiated by virtual photons, i.e., electron scattering. We have not studied this process yet in any systematic way. A statement expressing our intention to consider it, will appear in this year's research proposal. 
Energy-weighted sum rules for current densities in a non-relativistic many-body sys tem.

A number of energy-weighted sum rules have been discussed. The power of the excitation energy has been selected to correspond to the expressions appearing in the high-frequency limit of the corresponding response function describing the system. Both longitudinal and transverse transition operators have been considered. We shall not describe here any of the details of the functional structure of the results, but attempt only to summarize some of their qualitative features. What we are evaluating is effectively expectation values of current commutators or, somewhat more precisely, the commutator of a current operator with its own time derivatives. For the many-body case, these still contain sums over one-body terms but, in addition, two (or more) body terms appear. The one-body terms correspond to expressions similar to what we have reported before: the main feature is still the locality of the commutators, i.e., that they vanish when their position variables correspond to different points in space. The polynomial structure of the result in powers of the momentum transfer is exactly what we expect from general considerations. The two body terms lead to non-localities in the commutators. They are associated with correlation terms due to the presence of particle-particle forces, twobody terms in the current densities and the finite size of the particles. The correlation terms tend to disappear when the wavelength, determined by the reciprocal momentum transfer becomes shorter than the the appropriate correlation distance. The finite-size effects would also seem to disappear under similar circumstances, but the physical situation is in this case somewhat more subtle: after a certain point, intrinsic correlations representing the structure of the 
particle become relevant. This, in turn, is related to the fact that the sum rule must contain, also, terms representing the intrinsic excitation of the particles which, at some stage, becomes the dominant feature of the entire response function.

Charge-transfer excitations and the Cabibbo-Radicati sum rule.

We first briefly summarize the problem as stated in last year's proposal: the Cabibbo-Radicati sum rule was originally obtained from dispersion theory and current algebra. It applies to isospin one-half nuclei and charge-transfer transitions induced by general operators containing all orders of retardation. The same result has, on the other hand, been obtained for nuclei of any isospin by simple algebraic techniques applied to purely unretarded operators. This is surprising, since it implies that all retardation contributions vanish identically. We have calculated the most important contributions due to retardation and, as we had anticipated, found that they are not zero! The contradiction has its origin in the partial incompatibility between the conventional dispersion relations and the non-relativistic description of a nuclear system: the dispersion integral extends to high energies where the target cannot be assumed to move non-relativistically. In addition to the possible necessity for quantitative corrections, we clearly need a more consistent formulation of the problem. As a step in this direction, we have studied the conditions under which a restricted dispersion relation can be obtained, which includes lowest order retardation and is consistent with standard non-relativistic quantum mechanics. This program was completed in the form of a oncesubtracted dispersion relation subject to the condition that the energy transfer is limited to a range of approximately $100 \mathrm{MeV}$. Basica1ly, the problem 
is rather similar to the situation we discussed some time ago in connection with electromagnetic processes. The isospin-changing operators appearing in the present problem, however, introduce a number of differences which are quite significant when the ground-state isospin of the nucleus under consideration is different from zero. We note with some interest at this point, recent reports, from Michigan State University, of the possible observation in $p$, n reactions of states with $\mathrm{J}^{\pi}=\mathrm{I}^{-}$and isospin presumably one unit lower than that of the target. Confirmation of these reports will provide an additional motivation of our renewed interest in charge-transfer reactions. 
2B. (Levin)

\section{Consistency of approximate CCA wave function equations}

Item 3 under Year's Publications in last year's Progress Report (COO3235-87) is an article by J. M. Greben and F. S. Levin comparing model nuclear reaction calculations based on two approximate wave function schemes: the bound state approximation (BSA) to the channel coupling array (CCA) equations obtained using a channel permuting array and the familiar set of equations derived from the coupled reaction channel (CRC) method. This article has prompted an interesting controversy, to which was devoted much time, effort, correspondence, referencing and article writing, somelof the latter still in progress. The Greben-Levin article was attacked by N. Austern in a Physics Letter in which two claims were stated: first, that the BSA equations are "probably inconsistent" and second, that the CRC approach is superior to the BSA. Neither of these claims are correct, however: the BSA equations are consistent and neither of the CRC nor the BSA equations is known to be superior as determined via numerical comparison with exact calculations. Thus, the Austern claim of superiority is at present a matter of theology (ie, bias). These points were argued by Levin in a lengthy correspondence with Austern and established in articles by Levin and Li to appear in Physics Letters and in the Proceedings of the Ninth International Conference on the Few Body Problem. That the BSA equations are consistent now seems to be accepted unquestioningly.

\section{Derivation of the CRC equations}

Although the question of the consistency of the BSA equations is now settled, the Levin-Li claim that neither the BSA nor the CRC can be adjudged superior has not yet been accepted by Austern or other nuclear reaction theor- 
ists, despite lack of any numerical evidence. This is partly a matter of belief in what consitutes the optimal approximation when only a few bound states in each of on1y a few 2-cluster channels are employed as expansion bases. It is also partly due to an interesting result obtained by Kawai and Ichimura in a preprint which has been withdrawn following negative referee's reports. The result is a "derivation" of the CRC equations from the differential form of the CCA equations under approximations that, unlike the BSA itself, are not consistent with the CCA integral equations, ie, with the CCA boundary conditions. This derivation plus the attempts to derive the CRC equations from a particular n-particle scattering theory by Goldflam and Kowalski, as well as the desire to try to understand some aspects of an optimal set of approximate equations have motivated Levin and $\mathrm{Li}$ to enlarge on the Physics Letters article noted above. The meaning of "equivalent" in the statement that a particular wave function formalism is equivalent to a transition operator formalism has been clarified. In addition, a number of $n$-particle scattering theories have been examined in order to learn how the CRC equations may be obtained from them. This is straightforward for those wave function formalisms in which the wave function components are independent of the channel (or partitian labels): one can derive the CRC equations but only at the expense of connectivity. For the other kinds of wave function components, denoted "true componets" in last year's Progress Report, the CRC equations can only be derived by making ad hoc assumptions. This is consistent with the results of Goldflam and Kowalski. Finally, some aspects of CRC and the BSA related to diagonalization of the full Hamiltonian and its channel counterparts have been elucidated. This work, which is partly pedagogic and partly innovative is currently being written up in an article to be submitted for publication jointly with C. T. Li. 
The new multi-cluster, n-particle scattering theory

This new addition to the group of many-body scattering theories was discussed in last year's Progress Report and Renewal Proposal. An article on this theory was completed last January and has been accepted for publication in Annals of Physics. It derives the new equations, in both wave function component and transition operator form, and compares them with the corresponding BRS equations. Minimally coupled sets of equations are also obtained, as is a generalized distortion operator formalism peculiar to the new theory. Various research topics dealing with this theory were proposed in last year's Renewal Proposal, some of which have been investigated and are discussed below and others of which are in progress or under consideration. Two of the more important formal questions of interest concern unitarity and spurious solutions. A detailed investigation of the new transition operators has established using the methods of Polyzou that in the exact case, the usual discontinuity relation is satisfied, ie, the theory is unitary. This result need not hold when approximations are made. With regard to spuriosities, the key relation needed to ensure their absence and which holds for the threebody Faddeev wave function components does not occur in the new theory. This is a relation between each individual component and the Schrödinger wave function. As a result, spuriosities may occur. As in the case of those CCA equations obtained with a channel permuting array, this is no problem, since spuriosities are trivially identifiable and thus discardable for both exact and approximate solutions. In addition to these topics, work on two others was begun; one is complete. This latter is the development of a multiple scattering series representation for the new transition operators. The other topic concerns the structure of the equations for the case $n=4$, under the assumption of pair-wise interactions. In precursor form, there are 13 coupled 
equations when the four particles are assumed to be distinguishable and 3 coupled equations when all particles are identical. A detailed examination of the set of 13 coupled equations has revealed no simplifying features: they remain 13 complicated equations, the complexity arising from the fact that the only partition label not occuring is in the coupling terms defining selfcoupling. This is analogous to the Faddeev-Yakubovski and Alt-GrassbergerSandhas equations. Effort is thus being concentrated on the identical particle case, particularly because of our intent to study the nuclear four-body problem using the new equations.

Wave function formalisms in CCA theory

A paper on this topic, whose submission to Physical Review $C$ was noted in last year's Progress Report, has now appeared in print.

\section{Calculations using bound state approximations}

Work on this type of calculation, discussed in last year's Renewal Proposal, is still in the preparatory stage. It was decided not to carry out, those exact, separable-potential calculations (using the Doleschall 3-body code) that would be the theoretical "data" against which results of approximate calculations would be compared until the Koshel CRC code was modified and running on the IBM computer system at Brown University. The modifications have been difficult to implement because of the great complexity of the code, and although some progress in this direction has been made, much remains to be done. This work was begun by $\mathrm{Dr}$. Li, who successfully adapted the Doleschall code for use at Brown. Due to his other interests (see section 2C), $\mathrm{Dr}$. $\mathrm{Li}$ was not able to complete this work. Dr. Kuruoglu is continuing the work on this problem; it is one of his first undertakings as the new Research Associate in the group. 
Atomic and molecular bound state calculations

Exact, converged calculations for $\mathrm{H}_{2}^{+}$using the channel coupling array (CCA) equations have now been completed. They are extremely important not only as a means of establishing the validity of the CCA method as a bound state procedure, but also as a vehicle for testing the calculational procedure itself. As noted in previous Progress Reports, the following are general characteristics of the results of a limited Hilbert space basis diagonalization of the non-Hermitian, channel space Hamiltonian which appears in the CCA wave function equations: apparant convergence to incorrect results; simultaneous occurrence of both physical and unphysical molecular potential energy surfaces; energies which can lie lower than the exact ones and which can show an oscillatory behavior with increase in the basis size; and spurious solutions. All of these features have been seen in the $\mathrm{H}_{2}^{+}$calculations carried out via Hilbert space (global) basis diagonalizations. They raise several important questions, eg, what is the meaning of the relatively accurate gerade ground state energy surfaces; can the unphysical ungerade energy surfaces be eliminated and replaced by physical ones; and what role is played by the spurious solutions? These questions could be answered by even one converged CCA calculation. Such a calculation would also shed light on another question of fundamental importance which is at the heart of all CCA (and presumably many other n-particle scattering. theory) non-spurious bound state $(E<0)$ calculations. This concerns uniqueness of the solution. It has been proved, but only for $E>0$ (scattering), that in order to obtain a unique CCA solution, it is necessary to solve the full set of $N_{2}=2^{n-1}-1$ CCA equations corresponding to all 2cluster channels or partitions of the $n$ distinguishable particles. It is only inferred that the same set must be solved in order to obtain unique bound state solutions. While the question of the necessity of using the full set of. 
$\mathrm{N}_{2}$ coupled $\mathrm{CCA}$ equations in the bound state can only be answered negatively by a numerical calcuation, a CCA calculation involving all $\mathrm{N}_{2}$ equations which agreed with the exact Schrödinger equation results would at least provide a strong inference that the uniqueness requirement is the same for both positive and negative energies.

Converged calculations were carried out for $\mathrm{H}_{2}{ }^{+}$. using the finite element method (FEM). In contrast to the Hilbert space diagonalizations which involve globally defined functions, in the FEM local interpolation functions are used. A particular one of these latter functions will be non-zero only in one of the subdomains into which the full configuration or momentum space is divided Convergence is tested by decreasing the size of the subdomains and thus in-: creasing their number. Monotonic convergence was found, and a gerade ground state result was found that agreed with the exact Schrödinger equation result of Wind to better than 1 part in $10^{4}$. Similiarly, the unphysical ungerade result obtained by Krüger and Levin using a one-term diagonalization (the hydrogenic 1s state) was completely removed by use of the FEM: in its place was an accurate, physical ungerade surface. In general, the Schrödinger and CCA equations were solved simultaneously, and it was found that half of the CCA results agreed with the Schrödinger results to 10 decimal places for both gerade and ungerade states. The remaining CCA results were spurious (the sum of the two CCA components were zero for all internucleon separations) and did not affect the physical results. There were no unphysical results, and it is concluded that their occurrence in the previous limited Hilbert space basis diagonalizations is an artifact of that particular approximation procedure and not of the CCA method itself. Consequently, simple Hilbert space basis diagonalizations should be continued for more complex molecules; they may provide a basis for an atoms-in-molecule description of molecular structure. The cal- 
culations also clearly establish that for $\mathrm{H}_{2}^{+}$, both $\left(\mathrm{N}_{2}=2\right)$ channels are required to obtain unique results. The FEM itself has proved relatively easy to implement in the present bound-state case of $\mathrm{H}_{2}{ }^{+}$molecular ion structure. This ease and the accuracy of the results suggest that it could be a useful tool in the nuclear few-body problem, a point discussed in detail in the accompanying Renewal Proposal.

The results described above form a substantial portion of the $\mathrm{Ph}$. D. thesis of $\mathrm{Dr}$. Ford, who passed his doctoral oral in late August and then joined Xerox Corporation as an industrial post-doctoral fellow. A short letter on this work has been submitted for publication and two longer articles based on it are being prepared. 
2C. (Li)

\section{Consistency of the wave function components of the CCA theory}

In a recent article which appeared in Physics Letters, N. Austern has claimed that an approximate form of the wave function equations of the channel coupling array (CCA) theory of many-body scattering are probably inconsistent. Austern's conclusion is, however, incorrect. It is based on an ad-hoc formulation of the CCA wave function component equations which neglects the relevant boundary conditions. When these boundary conditions are imposed, ro inconsistencies are found. The details of analysis for this point are given in articles by Levin and $\mathrm{Li}$ to appear in Physics Letters and in the Proceedings of the Ninth International Conference on the Few Body Problem.

\section{Derivation of the CRC equations}

Various attempts to derive the coupled reaction channel (CRC) equations from the CCA or other particular n-particle scattering theory and the desire to try to understand some aspects of an optimal set of approximate equations have motivated Levin and $\mathrm{Li}$ to enlarge on the Physics Letters article noted above. A number of n-particle scattering theories have been examined in order to learn how the CRC equations may be derived from them. Besides, some structural aspects of CRC and the bound state approximation (BSA) to the CCA equations developed by J. M. Greben and F. S. Levin have also been elucidated. This work, done in cooperation with Prof..F. S. Levin, is currently being written up in an article to be submitted for publication. 
WKB-type äpproximation in angular momenta for central potential systems

This work is stimulated by the recent interest in applying the nonrelativistic quantum mechanics for central potentials to the $\mathrm{J} / \psi$ and other quark-antiquark $(q \bar{q})$ bound states as well as the desire to extend the previously developed calculational scheme based on Heisenberg matrix mechanics, as noted in last year's Progress Report, to more realistic physical systems.

First, we develop a large angular momentum expansion method within the framework of Heisenberg matrix mechanics for central potential systems. Then, via the introduction of a WKB-type analytic continuation procedure, the resulting asymptotic expansions for energy eigenvalues and other physical quantities, valid only for large angular momenta, are transformed into convergent expansions for essentially the whole range of the radial and orbital quantum numbers ( $n, 1$ respectively). From these, we obtain various orders of a WKB-type approximation in angular momenta which are most accurate for large 1 and small $\mathrm{n}$. In contrast, the corresponding orders of the usual WKB approximation obtained from asymptotic expansions in large radial quantum numbers are most accurate for small 1 and large $n$. For low-lying states of most central potential systems, the overall quality of the present WKB-type approximation in angular momenta is at least as good as or even better than the usual WKB approximation. Having only to solve at most a simple algebraic equation instead of the integral equations involved in the usual WKB approximation, the present WKB-type approximation in angular momenta is extremely useful for general central potential problems. Various examples including several quarkonium potentials as well as a short-range exponential potential are given to demonstrate the usefulness of the present method for theoretical discussion and as a viable alternative for numerical 
integration techniques: Besides the energy eigenvalues, we also make explicit calculations for wave function values at the origin as well as various E1 and MI transition matrix elements, which are of special important to quarkonium systems. Although the applications of the present method are made mostly to quarkonium systems, we also emphasize its applicability for central potentials such.as Yukawa and Woods-Saxon potentials frequently used in nuclear physics.

This work is currently being written up in an article to be submitted for publication. 
OTHER ACTIVITIES

\section{Ninth International Conference on the Few Body Problem}

The Ninth International Conference on the Few Body Problem was held in Eugene, Oregon 17-23 August 1980. Professor Levin was co-organizer of this conference along with Professor M. J. Moravcsik of the University of Oregon. These conferences have traditionally been of sufficiently small size (200300 participants) that the organizer(s) has not only headed the local organizing committee but has also functioned as the program committee, advised of course by the International Advisary Committee. This was true for the ninth conference, with Professors Levin and Moravcsik jointly sharing responsibility for program, format and organization. The majority of these activities were carried out in the period July 1979 - July 1980 at a separation of about 6,000 miles, Professor Levin being on leave of absence at Bonn University in West Germany during this time. Approximately $50 \%$ of his time for three months, and $25 \%$ of his time for another three months were devoted to conference activities. The apparant success of the conference was adequate justification for the time and effort put into its organization. In addition, Professors Levin and Moravcsik are co-editors of Volume II of the Proceedings, which contain the written versions of the plenary session talks. Volume II will be published in its entirety as an issue of the journal Nuclear Physics, and North Holland Publishing Company will also bring it out in a hardbound edition. External editorial work on the Proceedings was carried out after the conference ended.

\section{NATO Advanced Study Institutc}

In addition to the work on Few Body IX, Professor Levin also spent a large amount of time in the period November 1979-March 1980 drafting a pro- 
posal for NATO to sponsor an Advanced Study Institute on the topic "Many Body Scattering, Theory and Applications", to be held in July 1981. Nine other workers in this general area were contacted and agreed to be lecturers along with Professor. Levin in the event that NATO approval was secured. The proposal was submitted in mid-April and an unfortunate negative response was received in August, thus dashing hopes for this enterprise. In no way however, was the effort put into this activity a waste of time: it is obviously important to review one's research field in its various ramifications from tịme to time. In the present case, this turned out to be extremely advantageous, since this effort formed the core of the review work for Professor Levin's review talk at Few. Body IX on the topic "Some Recent Developments in n-Particle Scattering Theory". This review covered the two years from August 1978 and included, such diverse subjects as non-equilibrium statistical mechanics, numerical methods, time-delay theory and applications, nuclear reactions, atomic and molecular structure applications, and electron-hydrogen scattering. The writing of the review occupied most of the seven weeks prior to the beginning of the conference.

\section{Invited taiks}

The following invited lectures were given by members of the group:

"Few Body Model Calculations for Some Light Ion Induced Nuclear Reactions" (F.S. Levin, University of Tübingen, November, 1979).

"Many-body scattering phenomena, non-hermiticity and the structure of some simple atoms and molecules" (F.S. Levin, Institut de Physique Nucléaire, Orsay, November 1979)

"Many body scattering phenomena, nonhermiticity and the structure of some 
simple atoms and molecules" (F.S. Levin, Bonn University, January 1980)

"Many body scattering phenomena, nonhermiticity, and the structure of some simple atoms and molecules" (F.S. Levin, University of Kaiserslautern, February 1980).

"Many-Body Scattering Phenomena, Non-Hermiticity, and the Structure of Some Simple Atoms and Molecules" (F.S. Levin, Hahn-Meitner Institute, February 1980)

"Bound-State approximations in a three-body model of rearrangement collisions" (F.S. Levin, University of Surrey, March 1980)

"Many body scattering phenomena, and the structure of atoms and molecules" (F.S. Levin, University of Oxford, March 1980)

"Many body scattering phenomena, non-hermiticity, and the structure of some simple atoms and molecules" (F.S. Levin, Royal Holloway College, March 1980)

"Many Body Scattering Phenomena, Non-Hermiticity, and the Structure of Some Simple Atoms and Molecules" (F.S. Levin, Institut fur Radiumforschung and Kernphysik, Vienna, March 1980)

"Some Comments on Approximation Methods for Nuclear Reaction Analyses" (F.S. Levin, Central Research Institute for Physics, Budapest, March 1980)

"Many-body scattering phenomena, non-hermiticity, and the structure of some simple atoms and molecules" (F.S. Levin, University of Mainz, April 1980)

"Applications of Many-Body Scattering Theory to the Structure of Some Simple Atoms and Molecules" (F.S. Levin, Case Western Reserve University, May 1980)

"Some Comments on Bound-State Approximations for N-Body Scattering Systems" (F.S. Levin, invited paper presented at the European Symposium on Few Body 
Problems, Sesimbra, Portugal, June 1980)

"Many-Body Scattering Phenomena, Non-Hermiticity, and the Structure of Some Simple Atoms and Molecules" (F.S. Levin, Ruhr-Universität Bochum, June 1980)

"What Can Atomic and Molecular Physics Learn from Multiparticle Scattering

Theory?" (F.S. Levin, Hannover University, June 1980)

"A New Approach to the Calculation of Atomic and Molecular Energy Levels" (F. S. Levin, Max-Planck-Institut für Strömungsforschung, Göttingen, June 1980)

"Some Recent Developments in n-Particle Scattering Theory" (F.S. Levin, review paper presented at the Ninth International Conference on the Few Body Problems, Euguene, Oregon, August 1980)

"Nuclear Compton Scattering" (S. Fallieros, Michigan State University, June 1980) 


\section{P.ERSONNEL}

Professor S. Fallieros

Professor F. S. Levin

Research Associate Dr. C. T. Li (until. 31 August 1980)

Research Associate Dr. Z. Kuruoglu (from 25 August 1980)

Research Assistant W. K. Ford (until 31 August 1980)

Research Assistant R.. Kozack (from 1 June 1980) 


\section{YEAR'S PUBLICATIONS}

1. F. S. Levin, Wave Function Formalisms in the Channel Coupling Array Theory of Many-Body Scattering, Phys. Rev C 21, 2199 (1980)

2. F. S. Levin and C. T. Li, Consistency of the Wave Function Equations of Channel Coupling Array Theory, Phys. Letts. (in press)

3. A Multi-Cluster, n-Particle Generalization of the Three-Particle Faddeev Wave Function Equations, Annals of Physics (in press)

4. W. K. Ford and F. S. Levin, A Many-Body Scattering Theory Calculation of $\mathrm{H}+$ Potential Energy Surfaces (submitted for publication)

5. F. S. Levin, Some Comments on Bound-State Approximations for N-Body Scattering Systems, invited paper published in the Proceedings of the European Symposium on Few Body Problems (Sesimbra, Portugal, 1980), p.60

6. F. S. Levin, A Multi-Cluster, n-Particle Generalization of the Three Particle Faddeev Wave Function Components, contributed paper published in the Proceedings of the European Symposium on Few Body Problems (Sesimbra, Portugal, 1980), p.1.

7. F. S. Levin, Some Recent Developments in n-Particle Scattering Theory, invited paper to appear in Volume II of the Proceedings of the Ninth International Conference on the Few Body Problem.

8. F. S. Levin, A Multi-Cluster, n-Particle Generalization of the ThreeParticle Faddeev Wave Function Components, contributed paper published in The Proceedings of the Ninth International Conference on the Few Body Problem, Volume I, p. 15.

9. F. S. Levin and C. T. Li, Consistency of the Wave Function Components of Channel Coupling Array Theory, ibid. p. 16.

10. F. S. Levin and W. K. Ford, Convergence of a Channel Coupling Array Calculation of the $\mathrm{H}_{2}^{+}$. Ground State Energy, ibid, p. 17 
11. C. T. Li, Variational Principles for Particles and Fields in Heisenberg Matrix Mechanics (with A. Klein, and M. Vassanji), accepted for publication in Journal of Mathematical Physics.

12. S. Fallieros, Diamagnetism, Gauge Transformations and Sum Rules (with J. L. Friar) submitted for publication. 